\title{
Stroke and presence of patent foramen ovale in sickle cell disease
}

\author{
Constantina Aggeli ${ }^{1}$ Kali Polytarchou ${ }^{1,2}$ (1) $\cdot$ Yannis Dimitroglou ${ }^{1} \cdot$ Dimitrios Patsourakos $^{1} \cdot$ Sophia Delicou ${ }^{3}$. \\ Sophia Vassilopoulou ${ }^{4} \cdot$ Eleftherios Tsiamis $^{1} \cdot$ Kostas Tsioufis $^{1}$
}

Accepted: 9 February 2021 / Published online: 26 February 2021

(c) The Author(s), under exclusive licence to Springer Science+Business Media, LLC part of Springer Nature 2021

\begin{abstract}
Sickle cell disease (SCD) is an inherited monogenic hemoglobinopathy characterized by formation of sickle erythrocytes under conditions of deoxygenation. Sickle erythrocytes can lead to thrombus formation and vaso-occlusive episodes that may result in hemolytic anemia, pain crisis and multiple organ damage. Moreover, SCD is characterized by endothelial damage, increased inflammatory response, platelet activation and aggravation, and activation of both the intrinsic and the extrinsic coagulation pathways. Cerebrovascular events constitute an important clinical complication of SCD. Children with SCD have a 300-fold higher risk of acute stroke and by the age of 45 about $25 \%$ of patients have suffered an overt stoke. Management and prevention of stroke in patients with SCD is not well defined. Moreover, the presence of patent foramen ovale (PFO) increases the risk of the occurrence of an embolic cerebrovascular event. The role of PFO closure and antiplatelet or anticoagulation therapy has not been well investigated. Moreover, during COVID-19 pandemic and taking into account the increased rates of thrombotic events and the difficulties in blood transfusion, management of SCD patients is even more challenging and difficult, since data are scarce regarding stroke occurrence and management in this specific population in the COVID-19 era. This review focuses on pathophysiology of stroke in patients with SCD and possible treatment strategies in the presence of PFO.
\end{abstract}

Keywords Patent foramen ovale $\cdot$ Sickle cell disease $\cdot$ Stroke $\cdot$ Cerebrovascular event · COVID-19 - Antiplatelet therapy · Endothelial dysfunction

\section{Highlights}

- Patients with sickle cell disease (SCD) suffer from cerebrovascular events even from the early childhood. This has significant socioeconomic consequences, since it regards mainly young patients.

- Therapeutic management of SCD patients with cerebrovascular events is not well defined, even in most recent

Kali Polytarchou

kalipolyt@yahoo.gr

1 First Department of Cardiology, National and Kapodistrian University of Athens, Medical School, 114 Vasilisis Sofias, 11527 Athens, Greece

2 First Department of Cardiology, Henry Dunant Hospital Center, Athens, Greece

3 Thalassemia Unit, Hippokrateio Hospital, Athens, Greece

4 Department of Neurology, National and Kapodistrian University of Athens, Medical School, Athens, Greece
ASH guidelines, due to multifactorial pathophysiological mechanisms of stroke occurrence in this population.

- Presence of patent foramen ovale increases the risk of the occurrence of an embolic stroke. However, the role of PFO closure and antiplatelet/anticoagulation therapy has not been well investigated for these patients.

- Moreover, during the COVID-19 pandemic and taking into account the increased rates of thrombotic events and the difficulties in blood transfusion, management of SCD patients with stroke is even more difficult.

- This review focuses on pathophysiology and treatment strategies of stroke in patients with SCD and PFO.

\section{Introduction}

Sickle cell disease (SCD) is an inherited haemoglobinopathy caused by a single amino acid substitution at the sixth residue of the beta ( $\beta$ )-globin subunit (p. Glu6Val), which results in the formation of the characteristic haemoglobin 
$\mathrm{S}(\mathrm{HbS})$ [1]. In conditions of deoxygenation (when haemoglobin is not bound to oxygen), haemoglobin tetramers, which include two-mutant sickle $\beta$-globin subunits ( $\mathrm{HbS}$ ), can polymerize causing the erythrocyte to take a crescent or sickle cell shape [2]. These sickle cells are rigid and unstable, and also play a crucial role in acute and chronic SCD clinical manifestations. The increased adhesion of the sickle cells induces microvascular obstructions in capillaries resulting in blockage of blood flow with ischaemic/reperfusion injury [3]. SCD is a multi-system disorder that causes multiple organ damage $[3,4]$. Vaso-occlusion, haemolytic anaemia, and vasculopathy are the hallmark of SCD while organ damage is also associated with hypercoagulability and inflammation [5].

Sickle cell disease is characterized by endothelial damage, increased inflammatory response, platelet activation or aggravation and activation of both the intrinsic and the extrinsic coagulation pathways [3-6]. Cerebrovascular events constitute an important clinical complication of SCD. Children with SCD have a 300 -fold higher risk of acute stroke and by the age of 45 about $25 \%$ of patients have already suffered an overt stoke. Subclinical cerebrovascular disorder, confirmed by magnetic resonance imaging (MRI) scans, appears in another 10-20\% of SCD patients [7, 8].

Prevention, recognition and management of stroke in SCD patients are of incremental interest because of the young patients' age as well as the detrimental effect of stroke to quality of life, morbidity and mortality [9]. For this reason the 2020 American Society of Hematology (ASH) guidelines have suggested several recommendations for prevention and recognition of stroke in SCD patients as well as for treatment and rehabilitation [10]. However, as the guidelines pinpoint and according to a recently published systematic review, currently proposed management options have failed to prove significant benefits in the aforementioned goals when compared to improved standard care $[10,11]$.

The presence of a patent foramen ovale (PFO) is common in the general population and can be found in up to $25 \%$ of asymptomatic adults [12]. Paradoxical embolism through a PFO has been recognized as a cause of cryptogenic stroke in the general population and is present in a about $50 \%$ of those patients [13]. Thus in young patients with a cryptogenic stroke, thorough cardiovascular investigation aims to recognize PFO related stroke events and subsequently direct appropriate therapeutic interventions [14, 15]. PFO closure is superior to pharmacologic treatment when several clinical, echocardiographic and central nervous system imaging criteria are fulfilled [16, 17]. However, more data are needed before the implementation of such guidelines in specific populations such as in SCD patients [18].

Till now, it is not well understood how the presence of PFO affects the prognosis of SCD patients and thus investigation for the presence of PFO and modification in the management approach is not yet recommended. The scope of this review is to present the pathophysiology of stroke in patients with SCD and in patients with PFO with the view to recommend possible diagnostic and treatment strategies for SCD patients with presence of PFO.

\section{Cerebrovascular events in SCD patients}

\section{Epidemiology}

The occurrence of cerebrovascular events in patients with SCD is very high with severe socioeconomic consequences and constitutes the most important cause of neurocognitive deficits, reduced quality of life and increased morbidity among young SCD patients [10]. It is estimated that children with SCD sustain stroke 300-times more frequently than other children, in the form of silent cerebral infarctions, transient ischemic attacks, overt ischemic or hemorrhagic strokes $[4,8]$.

Clinically silent strokes are detectable accidentally in MRI by the age of 6 years in about $25 \%$ of children with SCD and this rate increases at $35 \%$ in young adults [19]. Moreover, about $25 \%$ of SCD patients by the age 25 will have had suffered at least one overt stroke [20]. Intracranial hemorrhage accounts for $3 \%$ and $10 \%$ of SCD patients in childhood and adulthood respectively [20].

For this special population, risk factors for ischemic stroke except for traditional risk factors, such as systemic arterial hypertension, renal disease, atrial fibrillation, hyperlipidemia and diabetes mellitus, include anemia, reduced $\mathrm{O}_{2}$ mean pressure (especially nocturnal hypoxia), reticulocytosis, and elevated lactate dehydrogenase or homocysteine levels [21].

\section{Pathophysiology}

Endothelial dysfunction and coagulation abnormalities are the main pathogenic mechanisms for a cerebrovascular event in patients with SCD. Hemolysis of sickle erythrocytes results in intravascular release of hemoglobin and haem, both of which promote oxidative stress, reactive oxygen species (ROS) production and severe reduction of nitric oxide (NO) production [22]. Moreover, intravascular hemolysis releases the enzyme arginase I and the asymmetric dimethylarginine (ADMA), both of which promote the uncoupling of NO synthase from NO production. As a result, there is a decreased NO production and increased ROS production and synthesis of polyamines, which facilitate cell proliferation and vascular remodeling $[4,22]$. The subsequent impaired vasodilatory microvascular response to NO could induce the expression of adhesion molecules from endothelial and blood cells and production of endothelin 1 . The increased 
expression of endothelial adhesion molecules (such as vascular cell adhesion protein 1, intercellular adhesion molecule 1, P-selectin, E-selectin, leukocyte surface antigen CD47 and $\alpha \mathrm{V} \beta 3$ integrin) result in leukocyte, erythrocyte and platelet adhesion, aggregation and activation and thrombus formation [23, 24]. In addition, activated endothelial cells produce inflammatory mediators, such as interleukin-1b, interleukin-6 and tumor necrosis factor leading to chronic inflammatory response $[4,23]$.

Endothelial dysfunction leads to vascular stenosis and obstruction, which, given the high cardiac output state in anemic SCD patients, leads to increased blood flow velocity in cerebral arteries as can be measured by transcranial Doppler (TCD) (Table 1) [25]. When total perfusion increases more than intrinsic mechanisms of the central nervous system can compensate, there is impairment of vasodilatory capacity and cerebral artery steal phenomenon is observed in areas supplied by stenosed arteries [26]. This in turn, leads to a reduction of oxygen supply in areas with cerebral stenosis, increasing vessel dysfunction, sickling of red blood cells and blood hyperviscosity, and thus promotes thrombosis of the cerebral arteries [27-29]. Recurrent episodes of thrombosis and subsequent thrombolysis exacerbate even further the endothelial dysfunction leading to accelerated cerebral artery dysfunction [23].

Microparticles derived from sickle cells hemolysis and endothelial dysfunction induce and maintain a hypercoagulable state in SCD patients through activation of both intrinsic and extrinsic coagulation pathway with the activation of tissue factor and factors VII, X, XI, XII [30]. It has been reported that SCD patients have increased plasma levels of prothrombin fragments, thrombin anti-thrombin complexes, D-dimers, fibrinogen and von Willebrand factor, while factors V, IX, XII and proteins C and S are decreased [30-32].

SCD patients have cerebral arteries stenosis and occlusion due to intima media proliferation, involving mostly internal carotid artery [3]. Subsequently, angiogenesis pathways are activated and collateral vessels are created around Circle of Willis to form a non-inflammatory vasculopathy named Moyamoya disease with a typical angiographic pattern $[33,34]$. This situation predisposes to recurrent ischemic strokes due to hypoperfusion and hemorrhagic stroke. Atherosclerosis or aneurysm of cerebral arteries is another cause of cerebrovascular events similarly to the general population. Finally, another common cause of stroke is paradoxical embolization. Patients with SCD have predisposition to venous thrombosis due to hypercoagulable state. In addition, during a vaso-occlusive crisis, due to bone marrow necrosis, the formation of fat emboli is common. In the presence of right- to- left shunt, embolization of thrombus or fat can result in ischemic stroke [21, 35].

\section{Diagnosis}

Diagnosis of stroke in SCD patients is similar to general population [36]. However, clinical signs and symptoms may be subtle and especially in pediatric population diagnosis can easily be missed. For that reason high clinical suspicion is at most importance.

Symptoms may include focal neurologic deficits, vision or language abnormalities, dizziness, vertigo, seizures, headache or migraines [37, 38]. Importantly, SCD patients often suffer from headache or migraine and it seems to be an association between severe headache and elevated mean cerebral flow velocities in TCD. In addition, severe headache may be the clinical symptom for cerebral sinus venous thrombosis, which may lead to stoke if diagnosis and anticoagulation therapy is delayed.

Computed tomography is the first imaging modality that can diagnose the presence of a hemorrhagic stroke in the acute phase. However, MRI with diffusion-weighted imaging is the preferred modality for assessment of ischemic stroke, even in the early phase [39]. Magnetic resonance angiography or venogram plays a crucial role in differential diagnosis of the cause of stroke (artery stenosis, venous thrombosis, embolic stroke or arterial aneurysm).

Last but not least, complete blood count, basic metabolic and electrolyte profile, blood glucose level, coagulation blood test and thrombophilic profile should be obtained for every patient with SCD presenting with signs and symptoms suggestive of a cerebrovascular event.

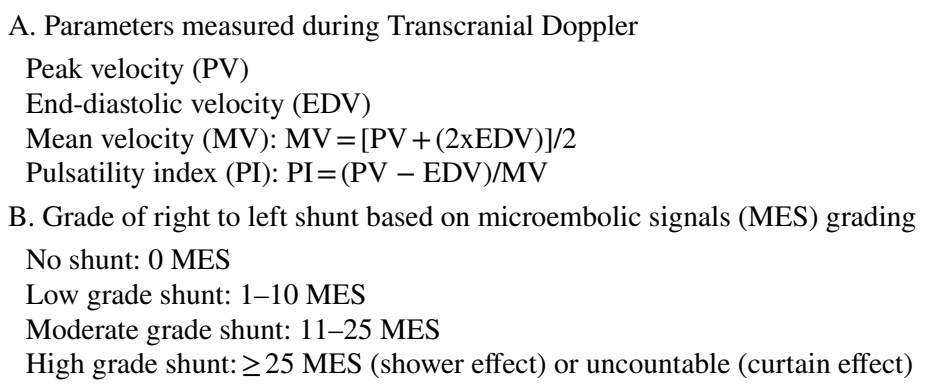




\section{Prevention and management}

Little evidence exists regarding the management of cerebrovascular events in patients with SCD for both primary and secondary prevention.

\section{Primary prevention}

The only approved strategy for primary prevention of stroke in patients with SCD is chronic blood transfusion and treatment of traditional risk factors, such as smoking cessation, hypertension, dyslipidemia or diabetes mellitus. Stroke Prevention in Sickle Cell Anemia ("STOP") Study, a multicenter randomized clinical trial which enrolled more than 2000 children with SCD, highlighted the role of TCD and the therapeutic value of blood transfusion in primary prevention of stroke for patients with SDC [40]. The role of TCD in identifying high risk patients for a cerebrovascular event has been established in recent ASH 2020 guidelines for SCD [10]. Annual TCD screening is recommended for children with $\mathrm{HbSS}$ or $\mathrm{HbS} \beta^{0}$ thalassemia (ages $2-16$ years) and is suggested for children who have compound heterozygous SCD other than HbSC and have evidence of hemolysis in the same range as those with HbSS [10].

Recent ASH guidelines recommend regular blood transfusion for at least a year with the goal of keeping maximum $\mathrm{HbS}$ levels $<30 \%$ and maintaining hemoglobin levels $>9.0 \mathrm{~g} / \mathrm{dL}$ to reduce the risk of stroke for children with $\mathrm{HbSS}$ or $\mathrm{HbS} \beta^{0}$ thalassemia (ages $2-16$ years) who have abnormal TCD velocities and live in a high-income setting (where regular blood transfusion therapy, typically every 3-4 weeks, is feasible to maintain the maximum $\mathrm{HbS}$ level $<30 \%$ and maintain the hemoglobin level $>9.0 \mathrm{~g} / \mathrm{dL}$ ) [10]. This is recommended also for children who have compound heterozygous $\mathrm{SCD}$ other than $\mathrm{HbSC}$, who have evidence of hemolysis in the same range as those with $\mathrm{HbSS}$, an abnormal TCD velocity, and live in a high-income setting [10].

The role of hydroxyurea in primary prevention of cerebrovascular stroke event has been investigated in the TCD With Transfusions Changing to Hydroxyurea (TWiTCH) Trial [41]. It is suggested that hydroxyurea treatment at the maximum tolerated dose can be considered to substitute for regular blood transfusions for children with SCD (ages 2-16 years), abnormal TCD results and without MRAdefined vasculopathy or silent cerebral infarct, who have been receiving transfusion therapy for at least 1 year, and are interested in stopping transfusion [10].

It is not known whether screening these patients for the presence of PFO with high risk features would be beneficial for primary embolic stroke prevention. Due to increased bleeding risk, the role of antiplatelet and anticoagulation therapy for primary prevention has not been investigated [6].

\section{Secondary prevention}

Exchange blood transfusion and hydroxyurea have been proven to be effective for secondary prevention of ischemic stroke in SCD patients [10, 41]. For children with HbSS or $\mathrm{HbS} \beta^{0}$ thalassemia and a history of prior ischemic stroke, ASH guidelines recommend blood transfusion for secondary stroke prevention aiming at increasing the hemoglobin $>9 \mathrm{~g} /$ $\mathrm{dL}$ at all times and maintaining the $\mathrm{HbS}$ level at $<30 \%$ of total hemoglobin until the time of the next transfusion [10].

Despite the presence of endothelial dysfunction, platelet activation and aggregation, increased inflammation and hypercoagulable state, concerning the role of antiplatelet and anticoagulation therapy in patients with SCD regarding primary and secondary prevention of stroke, scarce studies have been published supporting that there is increased risk for bleeding complications [6]. Until nowadays, aspirin is recommended for secondary prevention of stroke and the administration of heparin or warfarin is limited only for patients with paradoxical emboli and proven deep venous thrombosis, both based on recommendations for the general population [42]. However, ongoing trials are investigating the role of antiplatelets (eptifibatide and prasugrel) and anticoagulants (dalteparin and warfarin) as well as statins or new molecular anti-inflammatory or anti-oxidant agents in secondary prevention of stroke [6]. Crizanlizumab is a first-in-class, recombinant, humanized monoclonal antibody that blocks interactions between P-selectin and may play a crucial role in the prevention of sickle cell stroke [43].

\section{Patent foramen ovale and stroke in the general population}

\section{Epidemiological data and pathophysiology}

Patent foramen ovale is a communication between the right and the left atrium at the intra-atrial septum due to uncomplete fusion of the primum and secundum septum after birth. It is estimated that $25 \%$ of the general population has PFO, almost diagnosing accidentally with subtle clinical impact [44]. Nevertheless, the presence of PFO has been associated with paradoxical emboli. Subsequently, patients with a history of embolic stroke of unknown source should be evaluated by a cardiologist with transthoracic (TTE) or transoesophageal echocardiography (TEE) for possible embolic sources, such as the presence of right- to- left shunt through a PFO, aortic atheromatosis, cardiac masses (thrombus, myxoma or fibroelastosis) or atrial fibrillation.

The presence of PFO per se does not definitely set the diagnosis for paradoxical embolic stroke in a patient having suffered an ischaemic stroke. The patient history, clinical information and the cardiovascular and brain imaging data 
should be taken into account during consultation of a cardiologist, neurologist and radiologist. Certain clinical features, anatomical characteristics of the atrial septum and imaging features of brain MRI constitute the high risk patient for embolic stroke (Table 2). [44, 45].

\section{Diagnosis and management}

The combination of contrast TTE and contrast TCD could be the initial diagnostic approach for the evaluation mainly of young patients with ischemic stroke (Fig. 1) [46, 47]. Contrast TEE with intravenous administration of agitated saline and with simultaneous performance of Valsalva, with or without abdominal compression or cough maneuvers remains the most accurate method for diagnosing the presence of PFO [45, 47]. In addition, the anatomical characteristics and the size of foramen ovalis can be accurately defined by 2D and 3D TEE, as well as the aforementioned coexisting high risk anatomical characteristics that increase the likelihood of paradoxical emboli. The etiological relationship of embolic stroke with the presence of PFO should be carefully evaluated (Table 2) [44, 45].

Percutaneus PFO closure is the treatment of choice, but surgical closure is preferred when the anatomy of PFO is inappropriate for percutaneus closure. According to current recommendations for the general population, PFO closure is recommended for patients aged $<60$ years old, with recent ischemic stroke and presence of PFO with high risk criteria, as mentioned in Table 2, that is felt to be the most likely cause for stroke after etiological evaluation by a stroke expert (Fig. 2) [45, 48]. For patients not fulfilling the abovementioned criteria, clinical features suggesting paradoxical emboli, should be taken into consideration in addition to high risk characteristics of the patient, the PFO anatomy and the stroke imaging characteristics (Table 2) [45, 46].

\section{Patent foramen ovale in SCD patients with stroke}

Patients with SCD and stroke have been reported to have higher prevalence of PFO than the general population with stroke, although there are no prospective clinical trials that have studied the prevalence of PFO in individuals with SCD independently of stroke occurrence $[49,50]$. Moreover, SCD patients with PFO and right- to- left shunt may have increased risk for paradoxical embolism, since the pain they feel during a pain crisis may lead to increased endothroracic pressures, like when performing Valsalva maneuvers $[44,45]$. In addition to the hypercoagulable state and the high prevalence of arterial pulmonary hypertension in these patients, it seems that they are at high risk for stroke occurrence and recurrence $[35,50]$. Finally, they are young patients and stroke prevention must be a priority due to
Table 2 High risk features suggesting paradoxical embolism as the cause of stroke

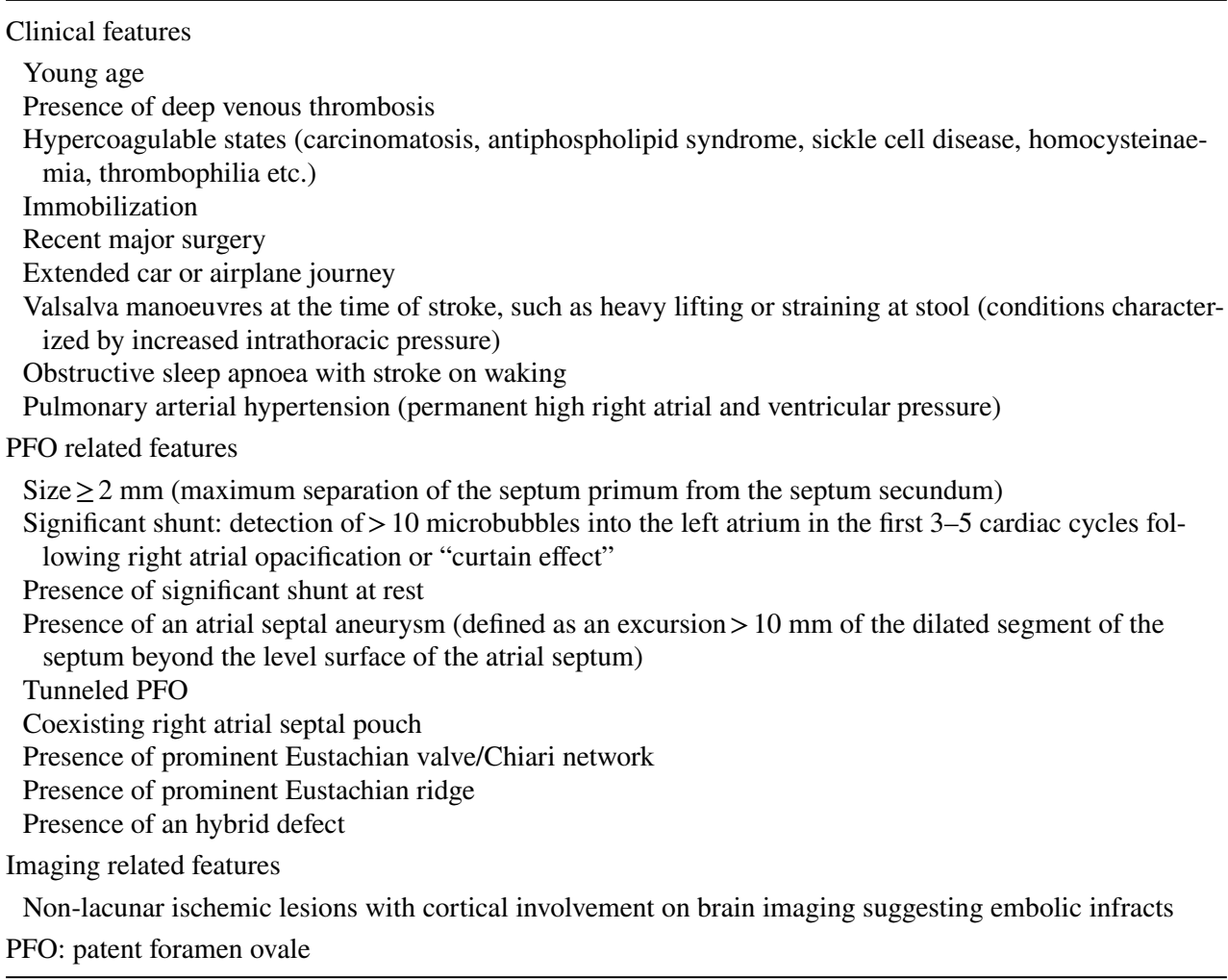



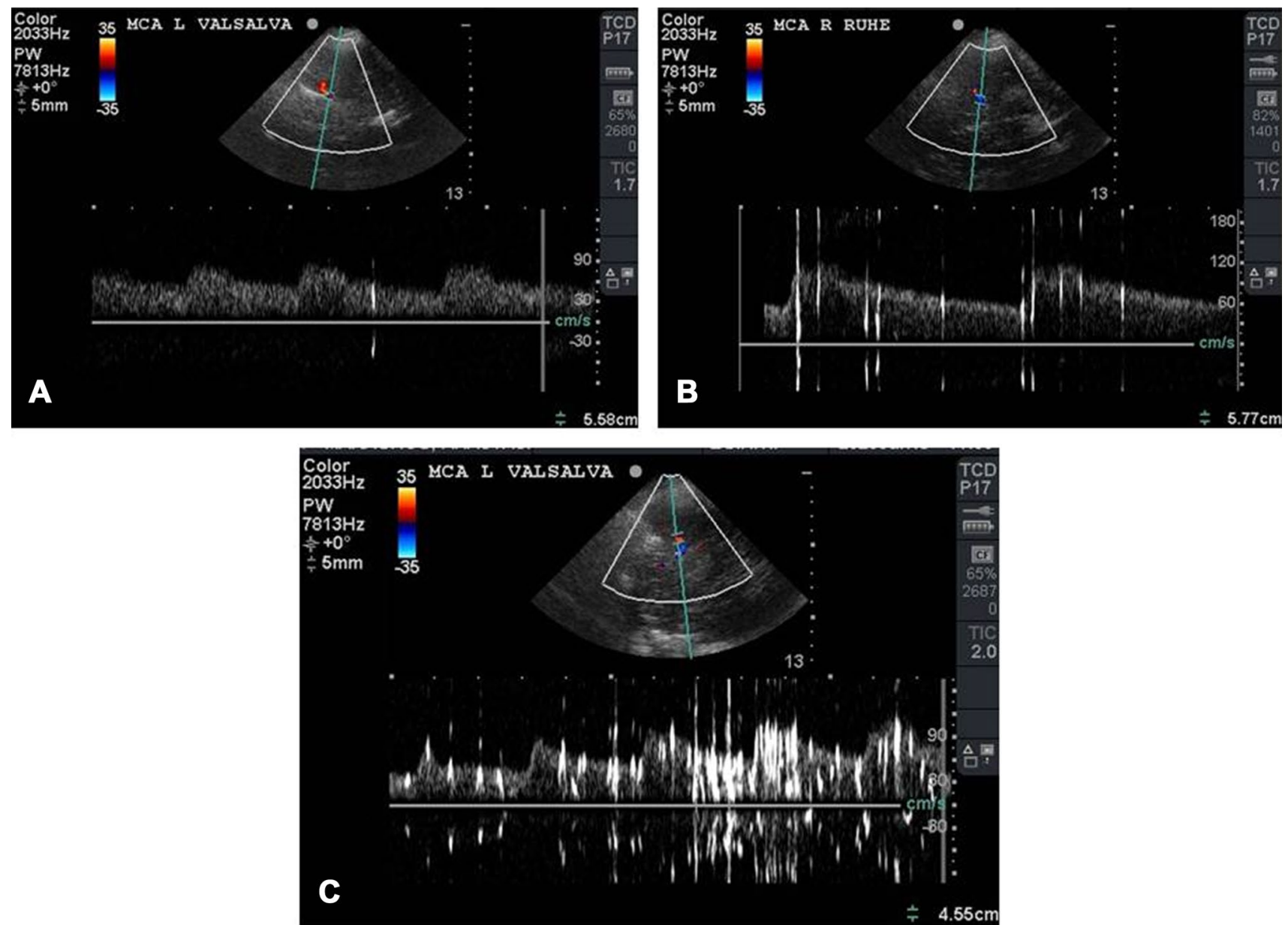

Fig. 1 Grade of right to left shunt based on microembolic signals (MES) grading. Transcranial Doppler of patients with a low grade shunt: 1-10 MES, b moderate grade shunt: 11-25 MES and c high grade shunt: $\geq 25$ MES or "shower" effect
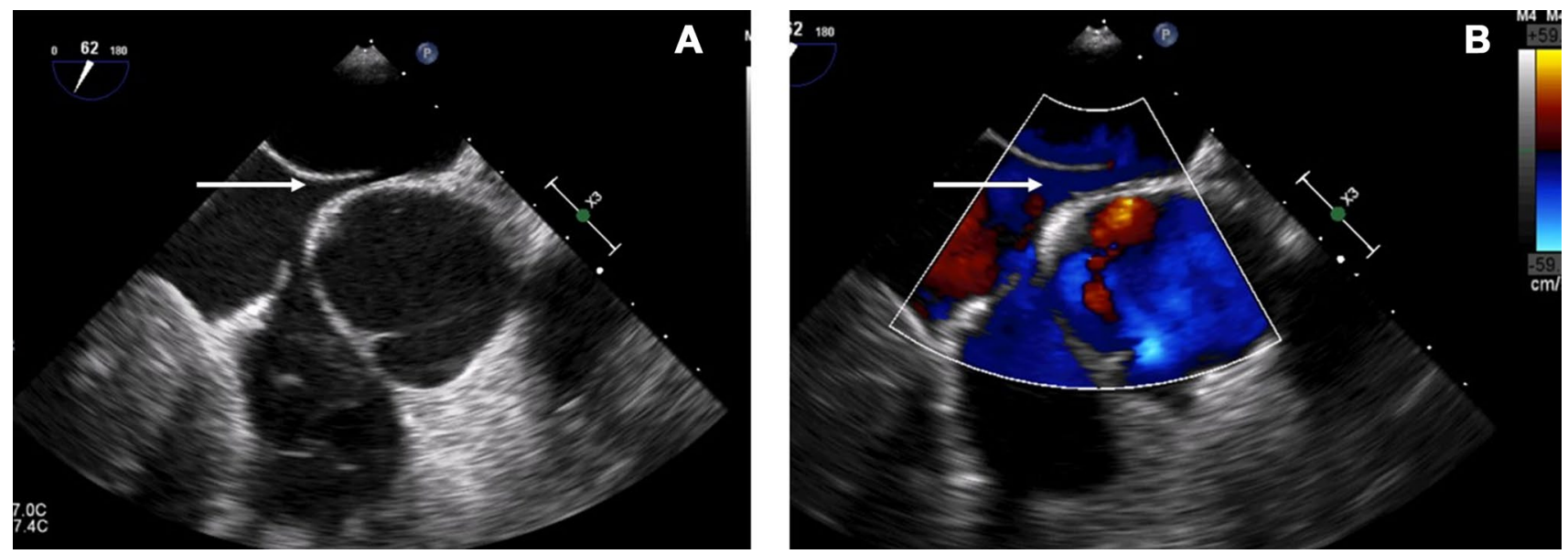

Fig. 2 a 2-D echocardiography reveals the presence of a tunneled patent foramen ovalis with high risk features (white arrows). b Color Doppler reveals significant shunt at rest 
severe physical (immobility, neurocognitive dysfunction), psychological and socioecomnomical consequences. Subsequently, the decision of PFO closure for these patients should be made taking into account all these parameters.

\section{Future perspectives}

The role of transcatheter closure of a PFO in patients with SCD has not been well investigated. However it may be effective in a portion of SCD patients having suffered an embolic stroke. The Risk of Paradoxical Embolism Score (RoPE score) has not been validated for SCD patients and its value has not been clearly defined in clinical practice. Recent European position for the management of patients with PFO suggests that in the presence of hypercoagulable state, deep vein thrombosis or pulmonary embolism, PFO closure may be considered when there is the need for only temporary anticoagulation therapy or when there is high risk for recurrence of stroke despite on anticoagulation therapy [45]. As mentioned before, patients with SCD are characterized by hypercoagulable state and are at increased risk for thrombosis. On the other hand the lifelong anticoagulation therapy increases the cerebral bleeding risk. Subsequently, PFO closure for patients with SCD having suffered an embolic stroke might be beneficial for secondary prevention and reduce the bleeding complications of anticoagulation therapy. Until recently, no study exists regarding the role of PFO closure in preventing a cerebrovascular event in patients with $\mathrm{SCD}$, only case reports have highlighted the gap in literature for these patients [4].

In the lack of recommendations, management of SCD patients having suffered an ischemic cerebrovascular event should be individualized. Cerebral MRI and magnetic angiography are very helpful with high diagnostic accuracy in differential diagnosis of an embolic stoke from an ischemic stroke due to underlying vasculopathy, like Moyamoya disease (Fig. 3). In the presence of a cerebral cardioembolic event, c-TEE should be performed (with Valsalva, abdominal compression or cough maneuver) in order to define the precise anatomy of fossa ovalis as well as the presence of right to left shunt. Transcranial Doppler should also be taken into account in order to proceed to the diagnostic and therapeutic approach (Fig. 1 and Table 1). Consultation of a neurologist, a hematologist, a radiologist and a cardiologist is very important in decision making for PFO closure and further treatment strategy for these patients.

It's uncertain whether the PFO closure should offer more or less confidence and safety in this patient population. However, taking into account the pathophysiology of cerebrovascular events in SCD, the increased endothoracic pressures during crisis, the increased bleeding risk while on anticoagulation therapy and the young age of these patients, it seems that PFO closure might be beneficial and interventional

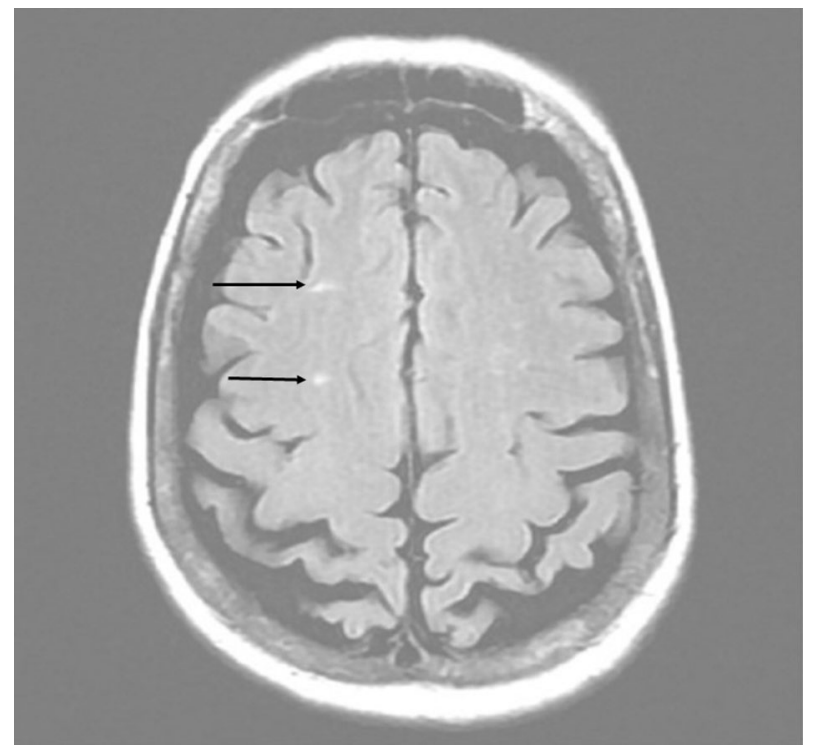

Fig. 3 Two high-intensity foci of ischemic origin located at the upper section of the right parietal lobe (black arrows) indicative of embolic stroke

approach should be preferred over conservative management.[51] Moreover, scarce evidence exists regarding optimal antiplatelet therapy after PFO closure for SCD patient and suggestion should be based on recommendations for the general population. In general, dual antiplatelet therapy with aspirin and clopidogrel is recommended for 3 to 6 months after successful PFO closure followed by single antiplatelet, preferred with clopidogrel $75 \mathrm{mg} /$ day, indefinitely. [51] Strong data should be reported on that issue.

In the COVID-19 era, strong evidence exists regarding the increased rates and higher mortality of thrombotic events and strokes [52]. Moreover, the lack of blood offer consists an important problem for patients at need for regular transfusion therapies, such as SCD patients. Data are scarce regarding stroke occurrence and management for SCD patients during the pandemic. However, this highlights the need for more evidence regarding anticoagulation and antiplatelet therapy beyond or in addition to blood transfusion and hydroxyurea for these patients.

\section{Conclusions}

Patients with SCD are at increased risk of stroke. The presence of a PFO increases the risk of stroke occurrence and recurrence in these patients. Decision for PFO closure in these patients should be made after multidisciplinary consultation of a cardiologist, a neurologist, a hematologist and a radiologist and taking into account the high risk features of the PFO, the stroke characteristics in brain imaging and 
the patients' ischemic and bleeding risk. More prospective clinical trials are needed for stroke prevention and treatment strategies in SCD patients.

Author contributions C.A. and K.P. have equally contributed to the article. C.A. and K.P. conceived of the presented idea and wrote the manuscript. Y.D., D.P., S.D., S.B., E.T. and K.T. read and thoroughly reviewed and commented on the manuscript.

\section{Compliance with ethical standards}

Conflict of interest All authors declare that they have no conflict of interest.

\section{References}

1. Bunn HF (1997) Pathogenesis and treatment of sickle cell disease. N Engl J Med 337:762-769

2. Brittenham GM, Schechter AN, Tom Noguchi C (1985) Hemoglobin $\mathrm{S}$ polymerization: primary determinant of the hemolytic and clinical severity of the sickling syndromes. Blood 65:183189. https://doi.org/10.1182/blood.v65.1.183.bloodjournal651183

3. Rees DC, Williams TN, Gladwin MT (2010) Sickle-cell disease. The Lancet 376:2018-2031

4. Kato GJ, Piel FB, Reid CD et al (2018) Sickle cell disease. Nat Rev Dis Primers 4:1-22

5. Naik RP, Streiff MB, Lanzkron S (2013) Sickle cell disease and venous thromboembolism: what the anticoagulation expert needs to know. J Thromb Thrombolysis 35:352-358

6. Ansari J, Moufarrej YE, Pawlinski R, Gavins FNE (2018) Sickle cell disease: a malady beyond a hemoglobin defect in cerebrovascular disease. Expert Rev Hematol 11:45-55. https://doi. org/10.1080/17474086.2018.1407240

7. Pavlakis SG, Bello J, Prohovnik I et al (1988) Brain infarction in sickle cell anemia: magnetic resonance imaging correlates. Ann Neurol 23:125-130. https://doi.org/10.1002/ana.410230204

8. Ohene-Frempong K, Weiner SJ, Sleeper LA et al (1998) Cerebrovascular accidents in sickle cell disease: rates and risk factors. Blood 91:288-294. https://doi.org/10.1182/blood.V91.1.288

9. Prussien KV, Jordan LC, Debaun MR, Compas BE (2019) Cognitive function in sickle cell disease across domains, cerebral infarct status, and the lifespan: a meta-analysis. J Pediatr Psychol 44:948-958. https://doi.org/10.1093/jpepsy/jsz031

10. DeBaun MR, Jordan LC, King AA et al (2020) American Society of Hematology 2020 guidelines for sickle cell disease: prevention, diagnosis, and treatment of cerebrovascular disease in children and adults. Blood Adv 4:1554-1588. https://doi.org/10.1182/ bloodadvances. 2019001142

11. Estcourt LJ, Kimber C, Hopewell S et al (2020) Interventions for preventing silent cerebral infarcts in people with sickle cell disease. Cochrane Database Syst Rev 4:CD012389

12. Lechat P, Mas JL, Lascault G et al (1988) Prevalence of patent foramen ovale in patients with stroke. N Engl J Med 318:1148 1152. https://doi.org/10.1056/NEJM198805053181802

13. Alsheikh-Ali AA, Thaler DE, Kent DM (2009) Patent foramen ovale in cryptogenic stroke: incidental or pathogenic? Stroke 40:2349-2355. https://doi.org/10.1161/STROKEAHA.109.54782 8

14. Mojadidi MK, Zaman MO, Elgendy IY et al (2018) Cryptogenic stroke and patent foramen ovale. J Am Coll Cardiol 71:1035-1043
15. Elgendy AY, Saver JL, Amin Z et al (2020) Proposal for updated nomenclature and classification of potential causative mechanism in patent foramen ovale-associated stroke. JAMA Neurol 77(7):878-886. https://doi.org/10.1001/jamaneurol.2020.0458

16. Lee PH, Song JK, Kim JS et al (2018) Cryptogenic stroke and high-risk patent foramen ovale: the DEFENSE-PFO trial. J Am Coll Cardiol 71:2335-2342. https://doi.org/10.1016/j. jacc.2018.02.046

17. Miranda B, Fonseca AC, Ferro JM (2018) Patent foramen ovale and stroke. J Neurol 265:1943-1949. https://doi.org/10.1007/ s00415-018-8865-0

18. Shatzel JJ, Daughety MM, Prasad V, DeLoughery TG (2018) PFO closure for secondary stroke prevention: is the discussion closed? J Thromb Thrombolysis 46:74-76

19. DeBaun MR, Armstrong FD, McKinstry RC et al (2012) Silent cerebral infarcts: a review on a prevalent and progressive cause of neurologic injury in sickle cell anemia. Blood 119:4587-4596

20. Debaun MR, Kirkham FJ (2016) Central nervous system complications and management in sickle cell disease. Blood 127:829-838

21. Hirtz D, Kirkham FJ (2019) Sickle cell disease and stroke. Pediatr Neurol 95:34-41

22. Kato GJ, Steinberg MH, Gladwin MT (2017) Intravascular hemolysis and the pathophysiology of sickle cell disease. J Clin Investig 127:750-760

23. Ansari J, Gavins FNE (2019) Ischemia-reperfusion injury in sickle cell disease: from basics to therapeutics. Am J Pathol 189:706-718

24. Proencą-Ferreira R, Brugnerotto AF, Garrido VT et al (2014) Endothelial activation by platelets from sickle cell anemia patients. PLoS ONE 9(2):e89012. https://doi.org/10.1371/journ al.pone. 0089012

25. Adams R, Nichols F, Carl E et al (1992) The use of transcranial ultrasonography to predict stroke in sickle cell disease. N Engl J Med 326:605-610. https://doi.org/10.1056/NEJM19920227326 0905

26. Prohovnik I, Hurlet-Jensen A, Adams R et al (2009) Hemodynamic etiology of elevated flow velocity and stroke in sickle-cell disease. J Cereb Blood Flow Metab 29:803-810. https://doi. org/10.1038/jcbfm.2009.6

27. Fields ME, Guilliams KP, Ragan DK et al (2018) Regional oxygen extraction predicts border zone vulnerability to stroke in sickle cell disease. Neurology 90:e1134-e1144. https://doi.org/10.1212/ WNL.0000000000005194

28. Václavů L, Petr J, Petersen ET et al (2020) Cerebral oxygen metabolism in adults with sickle cell disease. Am J Hematol. https ://doi.org/10.1002/ajh.25727

29. Hulbert ML, Ford AL (2014) Understanding sickle cell brain drain. Blood 124:830-831. https://doi.org/10.1182/blood-201406-582403

30. De Franceschi L, Cappellini MD, Olivieri O (2011) Thrombosis and sickle cell disease. Semin Thromb Hemost 37:226-236. https ://doi.org/10.1055/s-0031-1273087

31. Switzer JA, Hess DC, Nichols FT, Adams RJ (2006) Pathophysiology and treatment of stroke in sickle-cell disease: present and future. Lancet Neurol 5:501-512. https://doi.org/10.1016/S1474 $-4422(06) 70469-0$

32. Al-Awadhi A, Adekile A, Marouf R (2017) Evaluation of von Willebrand factor and ADAMTS-13 antigen and activity levels in sickle cell disease patients in Kuwait. J Thromb Thrombolysis 43:117-123. https://doi.org/10.1007/s11239-016-1418-4

33. Ito MT, da Silva Costa SM, Baptista LC et al (2020) Angiogenesis-related genes in endothelial progenitor cells may be involved in sickle cell stroke. J Am Heart Assoc 9(3):e014143. https://doi. org/10.1161/jaha.119.014143 
34. Guilliams KP, Fields ME, Dowling MM (2019) Advances in understanding ischemic stroke physiology and the impact of vasculopathy in children with sickle cell disease. Stroke 50:266-273. https://doi.org/10.1161/STROKEAHA.118.020482

35. Dowling MM, Quinn CT, Ramaciotti $C$ et al (2017) Increased prevalence of potential right-to-left shunting in children with sickle cell anaemia and stroke. Br J Haematol 176:300-308. https ://doi.org/10.1111/bjh.14391

36. Lawrence C, Webb J (2016) Sickle cell disease and stroke: diagnosis and management. Curr Neurol Neurosci Rep 6(3):27. https ://doi.org/10.1007/s11910-016-0622-0

37. Hines PC, McKnight TP, Seto W, Kwiatkowski JL (2011) Central nervous system events in children with sickle cell disease presenting acutely with headache. J Pediatr 159(3):472-478. https://doi. org/10.1016/j.jpeds.2011.02.009

38. Silva GS, Vicari P, Figueiredo MS, Junior HC, Idagawa MH, Massaro AR (2006) Migraine-mimicking headache and sickle cell disease: a transcranial Doppler study. Cephalalgia 26(6):678-683. https://doi.org/10.1111/j.1468-2982.2006.01092.x

39. Steen RG, Reddick WE, Mulhern RK et al (1998) Quantitative MRI of the brain in children with sickle cell disease reveals abnormalities unseen by conventional MRI. J Magn Reson Imaging 8(3):535-543. https://doi.org/10.1002/jmri.1880080304

40. Adams RJ (2000) Lessons from the stroke prevention trial in sickle cell anemia (STOP) study. J Child Neurol 15(5):344-349

41. Ware RE, Davis BR, Schultz WH et al (2016) Hydroxycarbamide versus chronic transfusion for maintenance of transcranial doppler flow velocities in children with sickle cell anaemia-TCD With Transfusions Changing to Hydroxyurea (TWiTCH): a multicentre, open-label, phase 3, noninferiority trial. Lancet 387:661-670

42. Klijn CJ, Paciaroni M, Berge E, Korompoki E, KõrvJ Lal A, Putaala J, Werring DJ (2019) Antithrombotic treatment for secondary prevention of stroke and other thromboembolic events in patients with stroke or transient ischemic attack and non-valvular atrial fibrillation: a European Stroke Organisation guideline. Eur Stroke J 4(3):198-223. https://doi.org/10.1177/239698731984118 7

43. Ataga KI, Kutlar A, Kanter J et al (2017) Crizanlizumab for the prevention of pain crises in sickle cell disease. N Engl J Med 376:429-439

44. Aggeli C, Verveniotis A, Andrikopoulou E et al (2018) Echocardiographic features of PFOs and paradoxical embolism: a complicated puzzle. Int J Cardiovasc Imaging 34(12):1849-1861. https ://doi.org/10.1007/s10554-018-1406-1
45. Pristipino C, Sievert H, D'Ascenzo F et al (2019) European position paper on the management of patients with patent foramen ovale. General approach and left circulation thromboembolism. Eur Heart J 40(38):3182-3195. https://doi.org/10.1093/eurheartj/ ehy649

46. Zhao H, Yue Q, Wang T, Wang L, Pang Z, Dong H, Yang J, Li Y, Li S (2019) Sensitivity of contrast-enhanced transthoracic echocardiography for the detection of residual shunts after percutaneous patent foramen ovale closure. Medicine 98(4):e14276. https ://doi.org/10.1097/md.0000000000014276

47. Takaya Y, Watanabe N, Ikeda M et al (2020) Importance of abdominal compression Valsalva maneuver and microbubble grading in contrast transthoracic echocardiography for detecting patent foramen ovale. J Am Soc Echocardiogr 33(2):201-206. https://doi.org/10.1016/j.echo.2019.09.018

48. Mas JL, Derex L, Guérin P et al (2019) Transcatheter closure of patent foramen ovale to prevent stroke recurrence in patients with otherwise unexplained ischaemic stroke: expert consensus of the French Neurovascular Society and the French Society of Cardiology. Arch Cardiovasc Dis 112(8-9):532-542. https://doi. org/10.1016/j.acvd.2019.06.002

49. Dowling MM, Lee N, Quinn CT et al (2010) Prevalence of intracardiac shunting in children with sickle cell disease and stroke. J Pediatr 156(4):645-650. https://doi.org/10.1016/j.jpeds .2009.10.012

50. Razdan S, Strouse JJ, Naik R et al (2013) Patent foramen ovale in patients with sickle cell disease and stroke: case presentations and review of the literature. Case Rep Hematol 2013:516705. https:// doi.org/10.1155/2013/516705

51. Giblett JP, Abdul-Samad O, Shapiro LM, Rana BS, Calvert PA (2019) Patent foramen ovale closure in 2019. Interv Cardiol Rev 14(1):34-41. https://doi.org/10.15420/icr.2018.33.2

52. Yaghi S, Ishida K, Torres J et al (2020) SARS2-CoV-2 and stroke in a new york healthcare system. Stroke. https://doi.org/10.1161/ strokeaha.120.030335

Publisher's Note Springer Nature remains neutral with regard to jurisdictional claims in published maps and institutional affiliations. 